\title{
Radioactive Seed Localization for Nonpalpable Breast Lesions in a Community Hospital Setting
}

\author{
James A. Wheeler ${ }^{1}$, Karlyn Harrod ${ }^{2}$, Fang Liu $^{2}$, Elizabeth Garber ${ }^{1}$, Lisa Grove-Narayan ${ }^{1} \&$ Adam Moore ${ }^{1}$ \\ ${ }^{1}$ Department of Radiation Oncology, Indiana University Health Goshen Center for Cancer Care, United States \\ ${ }^{2}$ Department of Applied and Computational Mathematics and Statistics, University of Notre Dame, United \\ States \\ Correspondence: James A. Wheeler, Department of Radiation Oncology, Indiana University Health Goshen \\ Center for Cancer Care, 200 High Park Avenue, Goshen, IN 46526, United States. E-mail: \\ jwheeler@goshenhealth.com
}

Received: December 8, 2015 Accepted: December 21, 2015 Online Published: January 8, 2016

doi:10.5539/cco.v5n1p25 URL: http://dx.doi.org/10.5539/cco.v5n1p25

\begin{abstract}
Purpose: To compare the positive margin rates for women with nonpalpable breast tumors whom had wire localization compared to radioactive seed localization in a small community hospital.
\end{abstract}

Introduction: Wire localized (WL) breast biopsies have been performed on patients with nonpalpable breast lesions for many years. Radioactive seed localization (RSL) offers advantages of patient comfort and scheduling convenience.

There is an extensive literature from large centers regarding the RSL technique. Little is known whether physicians performing these procedures in smaller community hospitals can achieve comparable negative margin rates as those performed with wire localization.

Methods: The thirty-six patients who underwent wire localized breast conserving surgery between September 18 , 2013 and December 10, 2014, were compared to the 48 radioactive seed localization patients resected between February 12, 2014 and December 18, 2014. The primary objective was to determine if the introduction of the radioactive seed localization technique significantly changed the positive margin rate.

Results: Two of 36 wire localized breast biopsied patients had positive margins, compared to 5 of 48 radioactive seed localization patients, a difference which is not statistically different. The specimen weight was larger for the patients treated with the RSL technique. There was a trend toward a higher positive margin rate with older patients.

Conclusion: The positive margin rate was similar between the wire localized and radioactive seed localized patients, and comparable to those in the published literature.

Keywords: Wire localized breast biopsies (WL), Radioactive Seed Localization (RSL), Breast conservation surgery (BCS)

\section{Introduction}

The American Cancer Society estimated that during 2015 there were 231,840 new cases of invasive breast cancer diagnosed among American women. Many of these new breast cancers are not palpable and consequently are detected with routine mammographic screening (the American Cancer Society, 2015). It is now well-established that the survival rate following breast conservation surgery and radiation therapy is equivalent to that following mastectomy (Darby et al., 2011). A recent Surveillance, Epidemiology, and End Results (SEER) registry study confirmed this is true even for younger women (Ye et al., 2015). The Society of Surgical Oncology and the American Society for Radiation Oncology recently published consensus guidelines on margin evaluation for patients being treated with breast conserving surgery and radiation therapy, establishing no tumor on ink as the standard for an adequate surgical margin (Moran et al., 2014).

For several decades, wire localized (WL) excisional breast biopsy has been the standard method to remove nonpalpable breast lesions. This involves placing a wire that exits through the patient's skin under ultrasound or mammographic guidance into the radiographic abnormality. The surgeon then uses the wire to direct the portion 
of the breast to be excised. This requires coordination between the radiologist, surgeon, and the operating room to minimize as much as practical the time between wire placement and subsequent excision. There is the potential for the wire to be displaced between the time of its image guided placement and subsequent removal in the operative suite. The surgical margins are frequently positive, which then requires an additional surgical procedure to remove more breast tissue to obtain clear margins (Cody \& Van Zee, 2015; McCahill et al., 2012). A recent report from the National Cancer Database including 316, 114 patients undergoing breast conserving surgery from 2004 through 2010, demonstrated roughly one-fourth of patients required a second procedure (Wilke et al., 2014).

Radioactive iodine-125 seed localization (RSL) is an attractive alternative. This typically involves placing a low intensity radioactive source under image guidance directly into the target breast lesion a few days prior to the planned breast conserving surgery. The surgeon then uses a radioactive probe to identify the target lesion in the breast. With this technique, the patient does not have a wire extending out from her breast. There is little likelihood for the marker to migrate a significant distance. There is no pressure to coordinate schedules between the radiology and the surgical staff.

The radiologist placing the iodine- 125 seed needs to be aware of potential pitfalls in optimal seed placement (Groudreau et al., 2015). The removed breast tissue requires special handling by the surgeon (Jakub et al., 2010) and pathologist (Graham et al., 2012). It also requires all of the involved personnel to pay careful attention to all of the radiation safety procedures and nuclear regulatory issues (Pavlicek et al., 2006).

There are several studies from large institutions in the United States and Europe comparing the positive margin rates between the wire localization and the radioactive seed localization techniques. A recent review article indicated positive margin rates are in the 5-25\% range for either technique (Lovrics et al., 2011). It remains to be shown whether or not similar results can be obtained using the RSL technique in a smaller community hospital.

\section{Method}

\subsection{Data Collection}

The study institution is an approximately 100 bed community hospital located in a rural community of 32,000. The hospital IRB gave approval to conduct this retrospective study. Through the tumor registry, 60 patients were identified who underwent breast conserving surgery between September 18, 2013 and December 10, 2014. Thirty-eight patients had nonpalpable tumors and had breast conserving surgery with wire localization. Two of these patients had benign disease, and were excluded from further analysis. These $36 \mathrm{WL}$ patients were compared with the RSL cohort. Some surgeons chose to not learn the RSL technique, so after the first patient was treated with the RSL technique on February 12, 2014, there were still patients who had wire localization.

In addition to the tumor registry, the Nuclear Regulatory Commission (NRC) requires detailed records regarding all patients undergoing the RSL procedure. Between February 12, 2014 and December 31, 2014, 58 patients with nonpalpable breast masses underwent breast conserving surgery using the RSL technique. Although all of the patients had previous needle biopsies, 10 patients had benign pathology and were excluded from the remaining analysis. This report concerns the remaining 48 RSL patients.

\subsection{Study Objectives}

The primary objective was to determine if the introduction of the RSL technique significantly changed the positive margin rate compared to the WL technique. Secondary objectives included determining if the likelihood of having a positive surgical margin depended on the patient's age, the breast volume, the weight of the breast specimen removed, or the volume of the operative bed determined on subsequent radiotherapy treatment planning CT images. Eleven of the $36 \mathrm{WL}$ patients either received their radiation therapy at another facility or otherwise did not receive it, so there was not a set of planning CT images to determine the volume of the operative cavity or the whole breast reference volume. One additional patient did not have contours for the operative bed. Similarly 13 of the RSL patients did not have radiation therapy at the study institution, so the volume of the operative cavity and the whole breast reference volume were only available for 35 RSL patients. Varian Eclipse version 11 software was used to compute the whole breast reference volume and the volume of the operative cavity based on the physician contours.

\subsection{Statistical Analysis}

The Fisher exact test was used to compare the margin positivity rate between the WL and RSL techniques. The p-value and the ratio between the RSL and WL techniques and the odds of having a positive margin were calculated. A logistic regression model was applied to explore the relationship between the likelihood of having a positive margin and the patient age, the whole breast reference volume, the weight of the breast specimen, and 
the volume of the operative bed. The t-test was performed to compare between RSL and WL regarding the minimum negative margin, the total treatment time from the initial biopsy to the date of the breast conservative surgery, and the amount of breast tissue removed by the weight of the specimen and by the volume of the operative bed as determined on the radiotherapy planning CT images. The data on the all the aforementioned variables were log-transformed before the t-test were performed to better satisfy the underlying normality assumption.

\section{Results}

\subsection{Patients}

Thirty-six patients underwent wire localization breast conserving surgery between September 18, 2013 and December 10, 2014. Forty-eight patients with DCIS or invasive breast cancer underwent resection using the RSL technique. Table 1 demonstrates the patient characteristics of both groups in terms of tumor stage and the patient age at the time of breast conserving surgery. As expected for patients with nonpalpable breast lesions, over half had stage I tumors. The patients in the RSL group had an average age of 62 compared to 65 for the WL patients. The median ages were 63 and 65 respectively.

Table 1. Patient characteristics

\begin{tabular}{lrr}
\hline STAGE & WL & RSL \\
\hline 0 & 10 & 6 \\
I & 21 & 30 \\
IIa & 4 & 9 \\
IIb & 1 & 2 \\
III & 0 & 1 \\
\hline AGE & WL & RSL \\
\hline$<50$ & 3 & 4 \\
$50-59$ & 10 & 16 \\
$60-69$ & 10 & 16 \\
$70-79$ & 8 & 8 \\
$80-89$ & 4 & 4 \\
$90-99$ & 1 & 0 \\
\hline Average age & 65 & 62 \\
\hline Pre-Op Chemo & 1 & 2 \\
Pure DCIS & 11 & 6 \\
Pure DCIS Average (mm) & 13.46 & 9.64 \\
DCIS Range (mm) & 1 to 47 & 1 to 50 \\
Invasive & 25 & 42 \\
Average Size (mm) & 13.4 & 14.2 \\
Invasive range (mm) & 6 to 25 & 3 to 40 \\
\hline
\end{tabular}

Note. $\mathrm{WL}=$ wire localization, $\mathrm{RSL}=$ radioactive seed localization

\subsection{Positive Margin Rates}

Two of the $36 \mathrm{WL}$ patients (5.5\%) had a positive margin compared to 5 of 48 RSL patients (10\%), with an estimated odds ratio $(95 \% \mathrm{CI})$ of $1.96(0.30,21.80)$ on the margin positivity between RSL and WL, but this difference was not statistically significant (p-value greater than 0.05 ).

\subsection{Covariates}

There was no statistically significant relationship between any of the examined covariates (patient age, whole breast reference volume, weight of the breast specimen, and volume of the operative bed) with the likelihood of 
having positive margins (all p-values greater than 0.25 ). There were also no statistically significant differences between RSL and WL on minimum negative margin (in $\mathrm{mm}$ ), or the total time (in days) from the initial biopsy to breast conservative surgery. There was a statistically significant difference between the WL and RSL patients in terms of the amount of breast tissue removed as measured either by the weight of the pathology specimens. The mean specimen weight was $33.24 \mathrm{~g}$ for the WL patients and $62.67 \mathrm{~g}$ for the RSL patients $(\mathrm{p}=0.02)$. There was no significant difference in the volume of the operative bed as seen on the radiation therapy treatment planning CT scans.

\section{Discussion}

Monica Morrow, MD reported the initial 6-month experience at Memorial Sloan-Kettering Cancer Center in which they had positive margin rates of $5.5 \%$ for their WL patients and $7.7 \%$ for their RSL patients (Murphy et al., 2013). They likewise reported no difference in the volume of the breast specimen removed by the surgeons using either the WL or RSL technique. Researchers from Canada likewise found no difference in the positive margin rates with WL (11.8\%) versus RSL (10.5\%) (Lovris et al., 2011). A study from the Netherlands Cancer Institute compared the positive margin rates of RSL (9\% RSL) with an alternative localization technique using $99 \mathrm{~m}$ technetium (10\%), with similar results (Van der Noodaa et al, 2015).

It is reasonable to suppose that the surgeon may be more generous in removing breast tissue from a patient with a larger breast compared to someone with a smaller breast. If this were the case, then one would expect a higher positive margin rate for those with a smaller breast. We examined the whole breast reference volume as determined by the radiotherapy planning CT imaging, but the margin positivity rate did not depend on the whole breast reference volume.

We also compared the volume of the operative cavity seen on the planning CT images with the weight of the breast specimen. Since the volume of the operative cavity decreases over time from resection, it was not surprising that the weight of the pathology specimen did not correlate with the operative bed volume as measured with the radiotherapy treatment planning CT images. The timing of the planning CT depended on whether the patient was treated with accelerated partial breast radiation (prior to chemotherapy), or upon completion chemotherapy (usually months later). Other factors such as developing a wound infection, the patient variability in the rate of wound healing, and the initial size of the seroma cavity also influenced how soon the patient was able to undergo radiation therapy treatment planning relative to the date of her breast conserving surgery.

The size of the minimum negative margin was not statistically significantly different with the WL and RSL techniques. There appeared to be a slight increase in the likelihood of obtaining a positive margin with increasing patient age, but this was not statistically significant. This is the opposite age dependence as was seen in the series from Seoul South Korea by Jung, where the patients younger than age 50 were more likely to require a re-excision for positive or close margins (Jung et al., 2012).

A recent study of 1,032 patients from China, of whom 209 had positive margins, reported by Jia, demonstrated that patients with Her-2 positive invasive breast cancers were more likely to have positive margins than patients with Her-2 negative tumors (Jia et al., 2014). Her-2 status was not evaluated in our study.

Since the RSL technique allows greater freedom in the scheduling of the breast conserving surgery, it was conceivable that the RSL technique might decrease the number of days separating the core biopsy from the breast conserving surgery. Excluding the single patient from the WL group who had pre-op chemotherapy, the median and the average number of days between the core biopsy and lumpectomy were 23 days and 30 days respectively. Excluding the 2 RSL patients who had preoperative chemotherapy, the median and average times from biopsy to lumpectomy were 27 and 46 days respectively. The range was 3 to 159 days for the WL patients and 6 to 256 days for the RSL patients. Other factors such as obtaining breast MRI imaging, choosing a breast surgeon, deciding between breast conserving surgery and mastectomy with or without reconstruction, as well as various patient preferences were likely far greater determinates of the interval separating the initial biopsy and breast conserving surgery than scheduling differences between the WL and RSL techniques. By way of comparison, the average time interval between the abnormal mammogram and the breast biopsy in 2014 was 9.8 days, with a range of 7.2 to 14.3 days.

Since our diagnostic radiologists were experienced in placing non-radioactive markers in the breast, learning the RSL technique was straightforward. The breast surgeons, similarly, were accustomed to using a gamma probe to identify the sentinel lymph node, and also found it relatively easy to use the probe to direct the excision of the relevant breast tissue. The RSL technique placed new demands on the pathology team to carefully extract the radioactive marker seed from the breast specimen and to provide the appropriate storage until the source was 
transported to the nuclear medicine department for appropriate disposition. The radiation safety officer also needed to provide the appropriate training of all of the individuals involved in handling the iodine- 125 marker seeds and caring for patients with an implanted radioactive source. The radiation safety officer must oversee the proper storage facilities and establish the appropriate documentation.

\section{Conclusion}

The RSL technique is a convenient alternative to WL of nonpalpable breast lesions. The radioactive localization seed can be placed under local anesthesia on a separate day than the breast conserving surgery. There is no pressure on the radiologist to place a marker wire immediately prior to the patient going to the operating room for breast conserving surgery. The patient does not have a wire sticking out of her breast that potentially could be displaced prior to surgery.

The RSL technique does place additional demands upon the nuclear medicine department, the radiologist, the breast surgeon, and the pathology department regarding the handling of the radioactive source and the appropriate documentation. Care needs to be taken by the surgeon and by the pathologist to not damage the radioactive marker seed. There is the potential for a seed to be lost either in the operative suite or in the pathology department. Training needs to be given to everyone involved with the care of the patient who has a radioactive seed in her breast and those handling the resulting surgical specimen. The patient can be assured that the radiation emitted from the time the source is placed in her breast until it is removed is not dangerous to her or to her family.

Although the published literature consists largely of large academic centers, the issues involved in conducting the RSL technique are the same for smaller community hospitals. Our experience should assure physicians practicing in community hospitals that they too can safely adopt the radioactive seed localization technique with the expectation of achieving the same low margin positivity rates as are obtained with conventional wire localization.

\section{Acknowledgement}

Victoria Owen, MD for reviewing the pathology slides when needed.

\section{References}

American Cancer Society. (2015). Cancer Facts \& Figures (p. 9). Atlanta.

Cody, H. S., \& Van Zee, K. J. (2015). Re-excision-The Other Breast Cancer Epidemic. N Eng J Med, 373(6), 568-569.

Darby, S., McGale, P., \& Correa, C. (2011). Effect of radiotherapy after breast-conserving surgery on 10-year recurrence and 15-year breast cancer death: meta-analysis of individual patient data for 10,801 women in 17 randomized trials. Lancet, 378, 1707-16. http://dx.doi.org/10.1016/S0140-6736 (11) 61629-2

Graham, R. P. D., Jakub, J. W., Brunette, J. J., \& Reynolds, C. (2012). Handling of radioactive seed localization breast specimens in the pathology laboratory. Am $J$ Surg Pathol, 36(11), 1718-1723. http://dx.doi.org/10.1097/PAS.ob013e318265c37f

Groudreau, S. H., Joseph, J. P., \& Seiler, S. J. (2015). Preoperative radioactive seed localization for nonpalpable breast lesions: technique, pitfalls, and solutions. RadiGraphics, 35(5), 1319-1334. http://dx.doi.org/10.1148/rg.2015140293

Jakub, J. W., Gray, R. J., Degnim, A. C., Boughey, J. C., Gardner, M., \& Cox, C. E. (2010). Current status of radioactive seed localization of nonpalpable breast lesions. American Journal of Surgery, 199, 522-528. http://dx.doi.org.10.1016/j.amjsurg.2009.05.019

Jia, H., Jia, W., Yang, Y., Li, S., Feng, H., Liu, J., ... Su, F. (2014). Her-2 positive breast cancer is associated with an increased risk of positive cavity margins after initial lumpectomy. World Journal of Surgical Oncology, 12, 289-298. http://dx.doi.org/10.1186/1477-7819-12-289

Jung W., Kang, E., Kim, S. M., Kim, D., Hwang, Y., Sun, Y., ... Kim, S. W. (2012). Factors associated with re-excision after breast-conserving surgery for early-stage breast cancer. $J$ Breast Cancer, 15, 412-419. http://dx.doi.org/10.4048/jbc.2012.15.4.412

Lovrics, P. J., Cornacchi, S. D., Vora, R., Goldsmith, C. H., \& Kahnamoui, K. (2011). Systematic review of radioguided surgery for non-palpable breast cancer. European Journal of Surgical Oncology (EJSO), 37, 388-397. http://dx.doi.org/10.1016/j.ejso.2011.01.018

Lovrics, P. J., Goldsmith, C. H., Hodgson, N., McCready, D., Gohla, G., Boylan, C., ... Reedjk, M. (2011). A 
multicentered, randomized, controlled trial comparing radioguided seed localization to standard wire localization for nonpalpable, invasive and in situ breast carcinomas. Ann. Sug Oncol, 18, 3407- 3414. http://dx.doi.org/10.1245/s10434-011-1699-y

McCahill, L. E., Single, R.M., Bowles, E.J.A., Feigelson, H.S., James, T.A., Barney, T., ... Onitilo, A. (2012, February 1). Variability in reexcision following breast conservation surgery. JAMA, 307(5), 467-475. http://dx.doi.org/10.1001/jama.2012.43

Moran, M., Schnitt, S., Schnitt, S. J., Guiliano, A. E., Harris, J. R., Khan, S. A., .. Morrow, M. (2014). Society of Surgical Oncology-American Society for Radiation Oncology consensus guideline on margins for breast conserving surgery with whole breast irradiation in stages I and II invasive breast cancer. Ann Surg Oncol, 21, 704-716. http://dx.doi.org/10.1245/S10434-014-3481-4

Murphy, J. O., Moo, T. A., King, T. A., Van Zee, K. J., Villegas, K. A., Stempel, M., ... Morrow, M. (2013). Radioactive seed localization compared to wire localization in breast-conserving surgery: initial 6-month experience. Ann Surg Oncol, December, 4121-4127. http://dx.doi.org/10.1245/s10434-013-3166-4

Pavlicek, W., Walton, H. A., Karstaedt, P. J., \& Gray, R. J. (2006). Radiation safety with use of I-125 seeds for localization of nonpalpable breast lesions. Academic Radiology, 13(7), 909-915. http://dx.doi.org/10.1016/j.acra.2006.03.017

Wilke, L. G., Czechura, T., Wang, C., Lapin, B., Liederbach, E., Winchester, D. P., \& Yao, K. (2014). Repeat surgery after breast conservation for the treatment of stage 0 to II breast carcinoma-a report from the National Cancer Data Base, 2004-2010. JAMA Surg., 149(12), 1296-1305. http://dx.doi.org/10.1001/jamasurg.2014.926

Ye, J. C., Yan, W., Christos, P. J., Nori, D., \& Ravi, A. (2015) Equivalent survival with mastectomy or breast conserving surgery plus radiation in young women aged $<40$ years with early-stage breast cancer: a national registry-based stage-by-stage comparison. Clinical Breast Cancer, 15, 390-397. http://dx.doi.org/10.1016/j.clbc.2015.03.012

Van der Noordaa, M. E. M., Pengel, K. E., Groen, E., van Werkhoven, E., Rutgers, E. J. Th, Loo, C. E., ... Peeter, V. (2015). The use of radioactive seed localization in patients with non-palpable breast cancer: a comparison with the radioguided occult lesion localization with 99m technetium. EJSO, 41, 553-558. http://dx.doi.org/10.1016/j.ejso.2015.01.022

\section{Copyrights}

Copyright for this article is retained by the author(s), with first publication rights granted to the journal.

This is an open-access article distributed under the terms and conditions of the Creative Commons Attribution license (http://creativecommons.org/licenses/by/3.0/). 